\title{
Experimental Results for Layered Targets Irradiated by Double Focal Spot
}

\author{
A. Aliverdiev ${ }^{a, b, c, *}$, D. Batani ${ }^{c, d}$, R. Benocci ${ }^{e}$, R. Dezulian ${ }^{e}$,

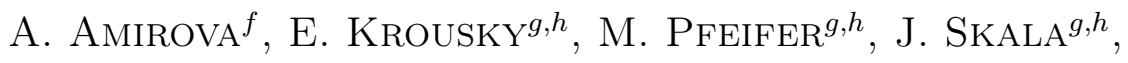 \\ R. DUdZAK ${ }^{g, h}$ AND K. JAKUBOWskA ${ }^{i}$ \\ ${ }^{a}$ IGRRE JIHT RAS, Pr. Shamilya 39A, 367030, Makhachkala, Russia \\ ${ }^{b}$ Dagestan State University, Gadjieva Str. 43A, 367025, Makhachkala, Russia \\ ${ }^{c}$ National Research Nuclear University MEPhI, Department of Plasma Physics, Moscow, Russia \\ ${ }^{d}$ University of Bordeaux, CEA, CNRS, CELIA (Centre Laser Intense at Applications), \\ UMR 5107, F-33405 Talence, France \\ e Universitá di Milano Bicocca, Piazza della Scienza, 20126 Milan, Italy \\ ${ }^{f}$ IP DFRC RAS, Yaragskogo Str. 94, 367003, Makhachkala, Russia \\ ${ }^{g}$ Institute of Physics ASCR, Na Slovance 1999/2, 182 21, Prague 8, Czech Republic \\ ${ }^{h}$ Institute of Plasma Physics ASCR, Za Slovankou 1782/3, 182 00, Prague 8, Czech Republic \\ ${ }^{i}$ IPPLM, Warsaw, Poland, Hery 23, 01-497 Warsaw, Poland
}

Doi: $10.12693 /$ APhysPolA.138.608

*e-mail: aliverdi@mail.ru

\begin{abstract}
We performed an experiment dedicated to study the interaction of a double-spot laser beam with aluminum foils and with foam-aluminum layered targets. The experiment was performed using the Prague PALS iodine laser working at $0.44 \mu \mathrm{m}$ wavelength and irradiance of a few $10^{15} \mathrm{~W} / \mathrm{cm}^{2}$. Shock breakouts for pure $\mathrm{Al}$ and for foam-Al targets have been recorded using time-resolved self-emission diagnostics and compared to results from numerical simulations. In the case of foam layered targets, we observed a spatial redistribution of the pressure and collision between two shocks originating from two spots. We also observed a significant delay of shock breakout in the shots with foam.
\end{abstract}

topics: laser-driven shocks, foams, impedance mismatch, shock collision

\section{Introduction}

Extreme states of matter are of interest to many branches of science. In recent years, the creation of samples with pressures of the order of tens megabar or more in laboratory conditions using laser-driven shock has become a standard tool in high-pressure physics [1].

Nevertheless, many phenomena still need to be studied, including shock dynamics in structured targets (micro- and nanostructured media) or dynamics of shock compression with multiple laser drivers. In this context, we have carried out an experiment to investigate the behavior and interaction of two shocks created inside a target by splitting the laser beam in two parts using a prism.

\section{Experiment and simulations}

The experiment was realized using the Prague Asterix Laser System (PALS) iodine laser [2]. The laser has wavelength of $0.44 \mu \mathrm{m}$ (the third harmonic of iodine laser) and is Gaussian in time with a full width at half maximum (FWHM) of about 300 ps.
In our experiment, the energy was ranging between 50 and $120 \mathrm{~J}$ and no phase plate was used in order to get smaller focal spots and higher intensities. By splitting the laser beam in two equal parts with a prism, we could obtain two focal spots with a diameter of about $70 \mu \mathrm{m}$ (FWHM) separated by about $200 \mu \mathrm{m}$, thus producing two different interaction points from which two laser-driven shocks originated.

The targets used in the experiment were either simple Al foils (10 $\mu \mathrm{m}$ thick) or double-layer targets made of $\mathrm{CH}_{2}$ foam (50 $\mu \mathrm{m}$ thick, on the laser side) and $\mathrm{Al}$ (10 $\mu \mathrm{m}$ thick, on rear side). Here we consider the experiment with the foam density of $50 \mathrm{mg} / \mathrm{cc}$.

As diagnostics, we used shock chronometry, i.e., we recorded and time-resolved the self-emission from the target rear side, allowing to measure the shock breakout time. A photographic objective was employed to image the target rear side onto a streak camera (Hamamatsu C7700) with an S-1 photocathode. A red RG60 filter before the streak camera cut out any $3 \omega$ light. A time fiducial was obtained by sending a small fraction of the incoming laser beam to the streak camera slit with an optical 
fiber. This was used to check the time of arrival of the laser beam on the target front side. A blue filter before the vacuum interaction chamber cut out $\omega$ and $2 \omega$ light. The interaction target front side was monitored by an X-ray streak camera (Kentech Low Magnification X-ray streak camera, by Kentech Instruments Ltd.) coupled to a $1024 \times 1024$ pixel 8-bit CCD. The X-ray streak was vertically positioned above the target.

Simulations of laser shock compression were carried out using the radiative hydrocode MULTI [3] in $1 \mathrm{D}$ and $2 \mathrm{D}$, with multigroup radiation transport coupled to Lagrangian hydrodynamics based on a fully implicit numerical scheme. We assumed LTE conditions. Equations of state were taken from SESAME [4] or calculated by MPQEOS [5]. The opacities were derived from $[6,7]$ or from a model implemented in the code SNOP [8-10].

\section{Results and discussion}

Experimental time-resolved images of rear-side self-emission obtained with the streak camera are shown in Fig. 1. Time flows from top to bottom. The signal on the upper left side of the image is the time fiducial indicating the arrival of the laser pulse on the front side of the target. The fiducial signal was installed using an optical fiber and tested on a series of calibration shots without a target. In the streak camera images, the fiducial arrives practically simultaneously to the main laser beam. However, in these shots, a set of filters was used
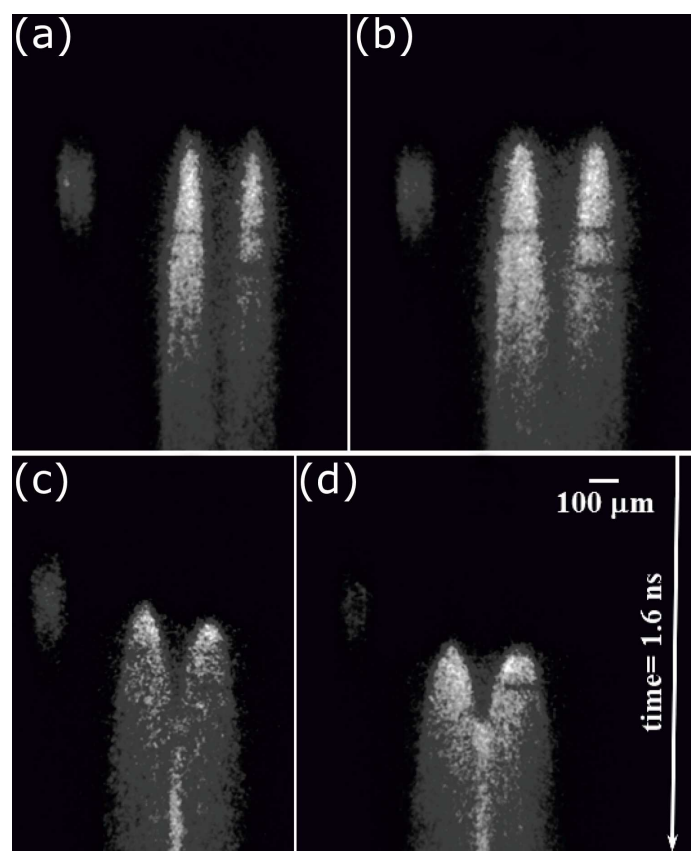

Fig. 1. Time-resolved images of the target rearside self-emission obtained with the streak camera (a) shot $\# 30142, E \cong 5 \mathrm{~J}$, simple $\mathrm{Al}$ target, (b) shot \#30141, $E \cong 115 \mathrm{~J}$, simple Al target, (c) shot $\# 30147, E \cong 50 \mathrm{~J}, \mathrm{Al}+$ foam $50 \mathrm{mg} / \mathrm{cc}$; (d) shot \#30148, $E \cong 115 \mathrm{~J}, \mathrm{Al}+$ foam $50 \mathrm{mg} / \mathrm{cc}$.
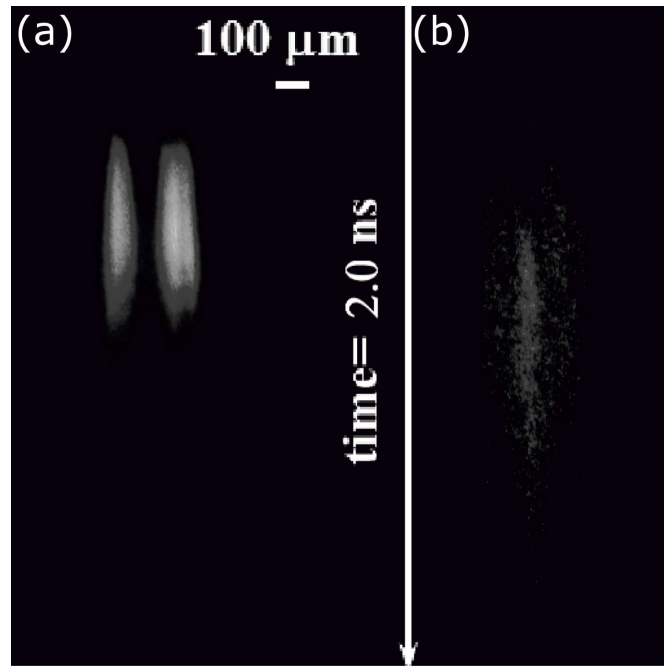

Fig. 2. X-ray streak-camera images for: (a) shot $30151, E \cong 50 \mathrm{~J}, \mathrm{Al}+$ foam $50 \mathrm{mg} / \mathrm{cc}$ with embed$\operatorname{ded} \mathrm{Au}$ nanoparticles; (b) shot $30147, E \cong 50 \mathrm{~J}, \mathrm{Al}$ + foam $50 \mathrm{mg} / \mathrm{cc}$.

to reduce the energy of the main laser beam incident on the visible streak camera. They introduced a delay in the fiducial of 180 ps relative to the main beam. Therefore, the laser beam arives in reality 180 ps before what is shown by the fiducial signal.

Figure 2 shows X-ray streak images for three shots. The X-ray streak camera, coupled to a pin-hole for space resolution, was looking at the target front side almost at $90^{\circ}$ with respect to the arrival of the laser beam.

Laser shots performed during the experiment are summarized in Table I. In each streak-images (Fig. 2), we observe two separated breakouts originating from the two focal spots. The time is given relative to the middle of fiducial (taking into account the correction due to glass filters).

Table I gives the difference between the arrival of the laser on the target front and the shock breakout time. A negative time of shock arrival means that the shock breakout takes place before the maximum intensity arrives on the target on the front side (here we used as references the maximum of the fiducial and the half-rise time of the shock breakout signal). The delay was corrected for 180 ps related to fiducial calibration, as mentioned earlier. The time at which the luminosity in the central region begins to rise is also presented in the table for the shots where this is clear.

Our data show a significant delay of shock breakout in the shots with foam. We can also observe that the size of the shock breakout signal for the foam-Al targets is larger than that observed for the pure $\mathrm{Al}$ targets. These data are easily explained as a consequence of the increased target thickness. This implies that more time is needed for the shock to breakout on the target rear side and that $2 \mathrm{D}$ effects become bigger implying a significant lateral expansion of the shock front. 
TABLE I

Time difference between shock breakout at the target rear and the arrival of laser beam on the target front. We report the shock breakout time (measured at half of rise) for the left $\Delta t_{\text {breakout }}^{\mathrm{L}}$ and the right $\Delta t_{\mathrm{breakou}}^{\mathrm{R}}$ spot as well as the time $\Delta t_{\text {cent. lumin. }}$ at which the luminosity in the central region begins to increase.

\begin{tabular}{|c|c|c|c|c|}
\hline Shot \# & 30141 & 30142 & 30147 & 30148 \\
\hline Target & $\mathrm{Al} 10 \mu \mathrm{m}$ & $\mathrm{Al} 10 \mu \mathrm{m}$ & $\begin{array}{c}\text { Foam } 50 \mathrm{mg} / \mathrm{cc} \\
50 \mu \mathrm{m} \\
+ \text { Al } 10 \mu \mathrm{m}\end{array}$ & $\begin{array}{l}\text { Foam } 50 \mathrm{mg} / \mathrm{cc} \\
50 \mu \mathrm{m} \\
+\mathrm{Al} 10 \mu \mathrm{m}\end{array}$ \\
\hline $\begin{array}{l}E_{3 \omega}[\mathrm{J}] \\
\text { (on target) }\end{array}$ & 115 & 50 & 50 & 115 \\
\hline $\begin{array}{l}\text { Total filter thickness } \\
\text { used before the laser beam }[\mathrm{cm}]\end{array}$ & 2.0 & 3.9 & 3.9 & 2.0 \\
\hline$\Delta t_{\text {breakout }}^{\mathrm{L}}[\mathrm{ps}]$ & -20 & 0 & 165 & 320 \\
\hline$\Delta t_{\text {breakout }}^{\mathrm{R}}[\mathrm{ps}]$ & 20 & 0 & 215 & 320 \\
\hline$\Delta t_{\text {cent. lumin. }}[\mathrm{ps}]$ & - & - & $\approx 610$ & 570 \\
\hline $\begin{array}{l}\text { Laser intensity on the target } \\
\text { (including } 40 \% \text { filter losses) } \\
{\left[\times 10^{15} \mathrm{~W} / \mathrm{cm}^{2}\right]}\end{array}$ & 3.0 & 1.3 & 1.3 & 3.0 \\
\hline $\begin{array}{l}\text { Simulation intensity } \\
\text { of laser }\left[\times 10^{15} \mathrm{~W} / \mathrm{cm}^{2}\right]\end{array}$ & 0.75 & 0.55 & 0.25 & 0.085 \\
\hline
\end{tabular}

Experimental data were compared to the results from numerical simulations performed with MULTI 1D. In the first step, we just simulated one focal spot to reproduce a single shock breakout. A significant difference was found since to reproduce the experimental shock breakout time, we needed to use an intensity of $(0.55 \div 0.75) \times 10^{15} \mathrm{~W} / \mathrm{cm}^{2}$ instead of the real intensity on the target $(1.3 \div 3) \times 10^{15} \mathrm{~W} / \mathrm{cm}^{2}$. Such a discrepancy was observed in previous experiments and was explained mainly as a consequence of the preheating induced by the hot electrons produced at such large laser intensities [10, 11]. Preheating causes a significant expansion of the target rear side resulting in a delayed shock breakout and in an apparent reduction of shock velocity. Then the "apparent" pressure is lower, implying that a lower laser intensity is sufficient to generate it. This type of results was correctly reproduced by hydro-simulations, using the code CHIC which self-consistently took into account the generation of hot electrons as a consequence of parametric instabilities, their propagation in the target and energy deposition and the consequent effects on hydrodynamics [11]. In this work, we did not follow such an innovative approach because we did not try to reproduce all details of experimental results but rather we studied relative changes occurring when the laser or target parameters were changed.

Our experimental results show that in the presence of the foam, the shock breakout time seems to increase rather than decrease when the pulse energy is increased (see Table I). This effect is indeed due to the preheating of the target by hot electrons, as considered in [10], because larger energies mean larger preheating effects due to hot electrons.
Another observed effect is the appearance of a bright region between the two spots for the shots with foams (see Fig. 1c, d) which is also present in the X-ray streak-camera image (see Fig. 2b). Such a bright region is not observed in the case of targets without the foam ablator (see Fig. 1a, b, Fig. 2a).

In order to qualitatively explain this phenomenon, we used 2D simulations. For simulating the experiment with a $2 \mathrm{D}$ code, we needed to assume axial symmetry. Therefore, each focal spot became a "ring" and we simulated the hydrodynamics produced by two concentric focal spots in the form of rings, observing what happens in the intermediate region. The results of these 2D-simulations for the simple and layered targets are presented in Fig. 3.

The impedance of aluminum is much higher than the impedance of foam and consequently after the reflection of the shock from the foam-metal interface, two shocks with the same pressure are generated: one of them is transmitted into the aluminum layer and the other is reflected back into the foam $[12,13]$. The reflected shocks continue to compress the foam-base and collide in the center producing a "long-living" central region with higher pressure. We can clearly see it in Fig. 4 which shows the spatial profiles of pressure (up) and temperature (low) for two times: (a) $550 \mathrm{ps}$, and (b) $650 \mathrm{ps}$. While after the breakout the rarefaction waves have already lowered the pressure and temperature in the main spots, the center formed as a result of the interaction of direct and reflected shocks in the metal-foam region remains compressed and hot.

It is also interesting to compare our results to the dynamics of shocks generated by a "doublespot" (same geometry, size and laser intensity) when 




Fig. 3. Temperature of the rear side obtained in 2D MULTI simulation for: (a) $10 \mu \mathrm{m} \mathrm{Al} \mathrm{target}$ with no foam, (b) target with $10 \mu \mathrm{m} \mathrm{Al}+50 \mathrm{mg} / \mathrm{cc}$ foam. Laser pulse with Gaussian time profile, duration $300 \mathrm{ps}$ (FWHM), wavelength $0.44 \mu \mathrm{m}$, and intensity $5.5 \times 10^{14} \mathrm{~W} / \mathrm{cm}^{2}$ (corresponding to the simulation laser intensity to have the breakdown time detected for the shot \#30142, pure aluminum target, total energy $50 \mathrm{~J}$ ). The artificial fiducial corresponding to the arrival of the main beam is shown on the right side.
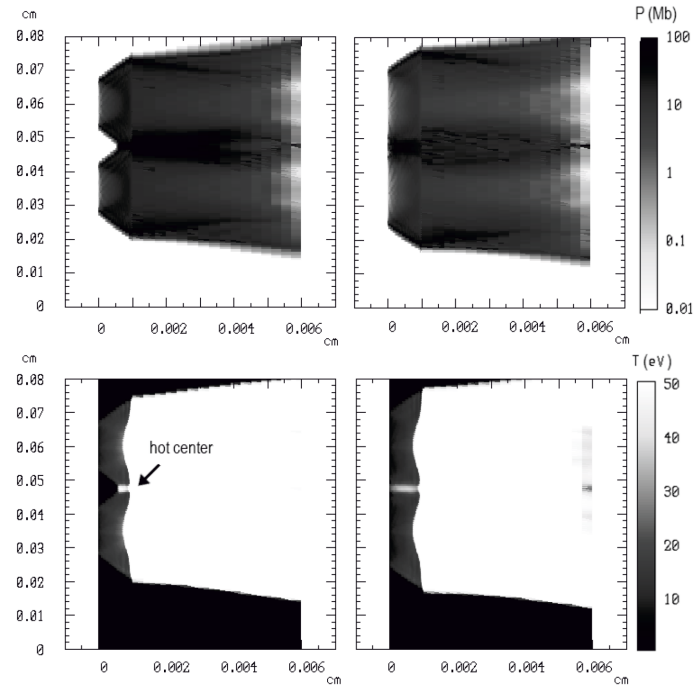

Fig. 4. Spatial shape of pressure (top) and temperature (bottom) for $t=0.55 \mathrm{~ns}$ (left) and $0.65 \mathrm{~ns}$ (right). The laser consisted of two rings (flattop, $100 \mu \mathrm{m}$ wide, $150 \mu \mathrm{m}$ distance), with a Gaussian time profile, a pulse duration of $300 \mathrm{ps}$ (FWHM), wavelength of $0.44 \mu \mathrm{m}$ and intensity of $5.5 \times 10^{14} \mathrm{~W} / \mathrm{cm}^{2}$ for the foam-Al target.

a gas jet is placed before the $\mathrm{Al}$ target [14, 15]. With the gas jet, the gas layer before $\mathrm{Al}$ is undercritical and the laser beam gets smoothed by ionization effects as it propagates through it. Therefore, the laser directly interacting at the front $\mathrm{Al}$ surface was already smoothed in a single spot. Consequently, the "collision" present in foam-layered targets was not observable in the gas jet experiment.

\section{Conclusion}

Our results indicate a significant delay in shock breakdown when the foam layer is present in the target. Also, the breakdown time on the laser pulse energy seems to increase when the laser energy increases. This unexpected behavior is likely due to the preheating of the target induced by hot electrons. Finally, we observed the collision between the two shocks produced by each focal spot, generating larger pressures in the collision region. Such pressure cumulation and redistribution can be interesting for applications in high energy density physics.

\section{Acknowledgments}

This work has been carried out within the framework of the EUROfusion Enabling Research Project: Enabling Research Project ENRIFE19.CEA-01 "Study of Direct Drive and Shock Ignition for IFE: Theory, Simulations, Experiments, Diagnostics Development" and has received funding from the Euratom research and training programme 2014-2018 under grant agreement No. 633053. The views and opinions expressed herein do not necessarily reflect those of the European Commission.

The work has also been supported by the European Union under the Laserlab programme, by the Competitiveness Program of NRNU MEPhI, Russia, and by the Czech Ministry of Education, Youth and Sports, projects LD14089 and LM2010014. A.A. is grateful to CNRS (France).

Finally, the authors are thankful to the PALS staff for help in running the experiments.

\section{References}

[1] D. Batani, Europhys. Lett. 114, 65001 (2016).

[2] K. Jungwirth, A. Cejnarova, L. Juha et al., Phys. Plasmas 8, 2495 (2001).

[3] R. Ramis, J. Meyer-ter-Vehn, J. Ramírez, Comput. Phys. Commun. 180, 977 (2009).

[4] SESAME: The LANL Equation of State Database, Eds. S.P. Lyon, J.D. Johnson, Group T-1, LA-UR-92-3407, Los Alamos National Laboratory, 1992.

[5] A.J. Kemp, J. Meyer-ter-Vehn, Nucl. Instrum. Methods Phys. Res. A 415, 674 (1998).

[6] M. Murakami, J. Meyer-ter-Vehn, R. Ramis, J. X-Ray Sci. Technol. 2, 127 (1990).

[7] A. Rickert, in: Final Report of Third International Opacity Workshop $\&$ Code Comparison Study, MPI fuer Quantenoptik, Garching (Germany), 1994. 
[8] K. Eidmann, Laser Part. Beams 12, 223 (1994).

[9] K. Eidmann, W. Schwanda, I.B. Földes, R. Sigel, G.D. Tsakiris, J. Quant. Spectrosc. Radiat. Transf. 51, 77 (1994).

[10] D. Batani, L. Antonelli, S. Atzeni et al., Phys. Plasmas 21, 032710 (2014).

[11] A. Colaïtis, G. Duchateau, X. Ribeyre, Y. Maheut, G. Boutoux, L. Antonelli, Ph. Nicolaï, D. Batani, V. Tikhonchuk, Phys. Rev. E 92, 041101(R) (2015).

[12] D. Batani, A. Balducci, W. Nazarov, T. Löwer, T. Hall, M. Koenig, B. Faral, A. Benuzzi, M. Temporal, Phys. Rev. E 63, 046410 (2001).
[13] A.A. Aliverdiev, D. Batani, L. Antonelli, K. Jakubowska, R. Dezulian, A.A. Amirova, G.M. Gajiev, M. Khan, H.C. Pant, Phys. Rev. E 89, 053101 (2014).

[14] R. Benocci, D. Batani, R. Dezulian et al., Plasma Phys. Contr. Fusion 50, 115007 (2008).

[15] R. Benocci, D. Batani, R. Dezulian et al., Eur. Phys. J. Special Topics 175, 65 (2009). 